\title{
Caracterización nutricional de trece variedades de pastos naturalizados de la región Amazonas
}

\author{
Nutritional characterization of thirteen naturalized grass varieties of the \\ Amazonas region \\ Neiser Galoc ${ }^{1}$, Hector V. Vásquez Pérez ${ }^{2}$, Wilmer Bernal ${ }^{3}$
}

\section{RESUMEN}

Las muestras de pastos fueron tomadas de diferentes lugares de la región amazonas con el objetivo de determinar materia seca (MS), proteína cruda (PC), fibra cruda (FC), extracto etéreo (EE), cenizas (CZA) y extracto libre de nitrógeno (ELN). Además, se determinó la concentración de minerales, calcio $(\mathrm{Ca})$, fosforo (P), energía bruta (EB), como fibra detergente neutra (FDN), fibra detergente ácido (FD) y digestibilidad in vitro. Las muestras se clasificaron de acuerdo a su naturaleza en gramíneas y Leguminosas. Se ha determinado la composición química de 6 variedades de gramíneas, 5 variedades de leguminosas y 2 variedades de hojas de subproductos agrícolas, encontrándose que el Zacate contiene mayores porcentajes en MS, ELN, FDN y FDA, mientras que el Yuyo resalta por su alto contenido de PC, EE y EB y con respecto a Ceniza, Ca y Digestibilidad resalta el pasto Ovillo y el pasto Nudillo es el que contiene el mayor porcentaje de P; mientras que le pasto grama azul presenta mayor contenido de PC dentro del grupo de las gramíneas. En el grupo de las leguminosas y hojas de sub productos agrícolas, la Hoja de Yuca es la que contiene mayor porcentaje de MS, EB y EE, mientras que la Lengua de Vaca es la que contiene mayor contenido de PC, asimismo podemos resaltar que la mayor concentración de minerales Ceniza, Ca y P es el Siso, mientras que en FC y FDA es la Retama la que contiene los mayores porcentajes en su composición.

Palabras clave: Análisis proximal; caracterización nutricional; pastos naturalizados

\begin{abstract}
Pasture samples were taken from different parts of the Amazonas region. The chemical composition was made by proximal analysis, in order to determine dry matter (DM), crude protein (PC), crude fiber (FC), ether extract (EE), ash (CZA) and nitrogen-free extract (ELN). In addition, the concentration of minerals, calcium (Ca), phosphorus (P), crude energy (EB), neutral detergent fiber (NDF), acid detergent fiber (FD) and in vitro digestibility were determined. The samples were classified according to their nature in grasses and legumes. In conclusion, the chemical composition of 6 varieties of grasses, 5 varieties of legumes and 2 varieties of leaves of agricultural by-products has been determined, finding that the Zacate contains higher percentages in MS, ELN, NDF and FDA, while the Yuyo stands out for its high content of PC, EE and EB and with respect to Ash, $\mathrm{Ca}$ and Digestibility highlights the grass Ovillo and grass Knuckle is the one that contains the highest percentage of P; while bluegrass grass presents higher PC content within the group of grasses. In the group of legumes and leaves of sub agricultural products, the Cassava Leaf is the one that contains the highest percentage of MS, EB and EE, while the Language of Cow is the one that contains the highest content of PC, we can also highlight that the Higher concentration of minerals Ash, $\mathrm{Ca}$ and $\mathrm{P}$ is the Siso, while in FC and FDA it is the Retama that contains the highest percentages in its composition.
\end{abstract}

Keywords: Proximal analysis; nutritional characterization; naturalized pastures.

\footnotetext{
${ }^{1}$ Bach. Neiser Galoc Bachiller en ingeniería zootecnista FIZAB-UNTRMA-A. neygalc@gmail.com

${ }^{2}$ M.Sc. Hector Vladimir Vásquez Pérez-hvasquez@untrm.edu.pe

${ }^{3}$ M.Sc.Wilmer Bernal Mejía-wilmer.bernal@untrm.edu.pe
} 


\section{INTRODUCCIÓN}

El forraje proveniente de los campos naturales de pastoreo constituye el pilar de la supervivencia de las sociedades andinas que desarrollaron prácticas y estrategias ganaderas para aprovechar estos recursos. Investigadores afirman que hoy en día se encuentran altamente degradados debido a un sobrepastoreo y a un uso excesivo de su materia leñosa, de hecho, se encuentran zonas objetivamente en proceso de desertificación, relacionado con el uso ganadero y también con la actividad meramente agrícola. La presencia de extensas áreas cuyas condiciones agroclimáticas (salinidad de suelo y agua, altura, frío, aridez) constituyen serias limitantes para la agricultura, han favorecido la aparición y permanencia de sistemas de producción animal dependientes del uso de praderas naturalizadas. La vegetación naturalizada representa la base alimenticia exclusiva del ganado en las zonas sobre los $4000 \mathrm{msnm}$ y en aquellas con precipitaciones anuales inferiores a $350 \mathrm{~mm} / \mathrm{año}$, constituyendo un componente importante de su dieta (Genin et al., 2006).

Los pastos naturalizados soportan por décadas dotaciones excesivas de sequias y manejos inadecuados, que condujeron a pasturas resistentes al pisoteo, pero de baja producción. La importancia de los forrajes, como alimento de los hatos ganaderos, es una base primordial del desarrollo social y económico; los pastos ayudan a satisfacer las demandas en alimentos tan esenciales como la carne y leche y es fuente fundamental de generación de mano de obra e ingreso (Vera et al., 2009).

La ganadería con uso de pastizales naturalizados constituye una de las actividades económicas más importantes del espacio andino, los pastos, tanto naturalizados como cultivados, constituyen la base de la alimentación animal, mantienen al $84 \%$ de la ganadería nacional, sin embargo, se estima que solamente $9.5 \%$ de la vegetación naturalizada es de condición buena y por lo menos el $60 \%$ es de condición pobre (Rodríguez et al., 1986).

La mayoría de pastos naturalizados altos andinos están en manos de comunidades campesinas. Estos pastos difieren en valor nutritivo, capacidad de carga y productividad, lo cual da lugar a diferencias en la cantidad de leche y carne producida. Los pastos naturalizados soportan 0.37 Unidad animal (UA)/ha/año que producen un promedio $25.3 \mathrm{~kg}$ de carne y 33.4 litros de leche por hectárea para venta al mercado. En tanto que los pastos cultivados soportan 1.5 UA/ha/año, producen 900 litros de leche se obtienen mayores resultados cuando se trabaja mediante asociaciones de pastos nativos con pastos cultivables (Flores et al., 2008).

Los pastos, además de ser la fuente alimenticia más económica de la producción ganadera, constituyen la base de la dieta alimenticia del ganado bovino, suministran la mayor parte de los elementos nutritivos para la producción de leche y carne ; cerca del $65 \%$ de la agricultura nacional en el Perú depende de los recursos genéticos nativos y naturalizados, como las papas, el maíz, el camote, los granos andinos (quinua, kiwicha, caigua), los frutales y entre otros; así mismo, cerca del 95\% de la ganadería nacional depende de los recursos forrajeros nativos, como los pastos naturales alto andino y algarrobales de la costa norte (Brack, 2004). 
En el Perú la mayor población de bovinos está en manos de pequeños productores, de 1745773 UA; 846829 crían bovinos, es esencial el manejo de pastos naturalizados en las partes alto andinas del país para reservar las praderas naturales y desarrollar esta actividad, ya que tienen una gran capacidad para reducir la escorrentía e incrementar la infiltración del agua en el suelo para así producir forrajes para la alimentación del ganado. Sin embargo, ante esta situación, es necesario realizar prácticas de manejo adecuado de los pastos naturalizados, para recuperar su capacidad productiva, mejorar la cobertura vegetal, disminuir la escorrentía y la erosión de los suelos, e incrementar la infiltración y la recarga de los acuíferos. Muchas comunidades han iniciado trabajos de manejo de los pastos naturales, logrando recuperar la capacidad productiva y la condición óptima de los pastizales. Además, al manejar adecuadamente los pastos naturalizados, sin afectar su condición y productividad, evita la erosión de los suelos y mejora la capacidad de recarga hídrica (Rivera et al., 2008).

En la región Amazonas según el censo agropecuario el $80 \%$ de la población se dedican a la crianza de ganado vacuno, pero el problema principal que se enfrentan cada productor es la deficiencia de pasto que no soportan a la estacionalidad climática esto conlleva a una baja rentabilidad de producción de leche y carne, para ello se busca dar una alternativa, buscando pastos que sean resistentes a estos problemas que presentan el productor (Oliva et al., 2015)

\section{MATERIAL Y MÉTODO}

Las muestras se recolectaron de las provincias de: Rodríguez de Mendoza (Omia, Lima bamba,
Huambo, Cochamal); Bogará (San Carlos, Famrre, Cuispes, Agua Dulce, Poma cochas, Suyubamba); Chachapoyas (Chachapoyas capital, Pipus, Levanto, Caclic, Huancas, Molino pampa); Luya (Luya capital, Trita, Cohechan).

\section{a) Métodos}

Se utilizó el método inductivo, para evaluar la composición química de los pastos naturalizados y el método analítico para evaluar y analizar la composición nutricional de cada muestra.

Las técnicas utilizadas han consistido en recoger muestras de diferentes lugares de la provincia de Bongará (San Carlos, Fanrre, Cuispes, Poma cochas, Suyubamba); Chachapoyas (Chachapoyas, Pipus, Levanto, Caclic, Huancas, Molinopampa); Luya (luya, Trita, Cohechan); Rodríguez de Mendoza (Omia, Limabanba, Huambo, Cochamal)

Los métodos que se tuvo en cuenta para evaluar los factores de evaluación se tuvieron en cuenta las informaciones de los productores, las edades de crecimiento después que ha sido pastoreado

\section{b) Procedimiento}

Los procedimientos de caracterización bromatológica que se siguió en el presente trabajo de investigación, se detallan a continuación.

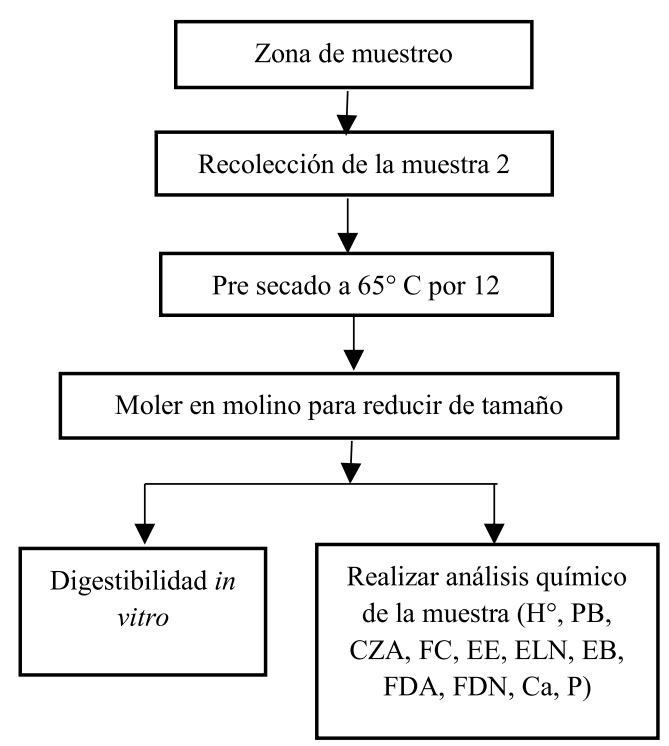




\section{c) Selección de las zonas a muestrear}

Las zonas a muestrear se seleccionaron de acuerdo a la producción y disponibilidad de sus forrajes más relevantes.

\section{d) Recolección de muestras}

Se recolectaron las muestras en 04 provincias, 18 distritos de la Región Amazonas. Para determinar los lugares de muestreo primero se realizó un estudio de línea de base, donde se determinó los lugares más representativos dentro de la región que predominan las muestras a ser recolectadas, cada muestra se recolectó con tres repeticiones, con ayuda de un cuadrante de tubo plastificado de $1 \mathrm{~m}^{2}$ la muestra se cortó a unos $5 \mathrm{~cm}$ del suelo en forma manual con un osino, el tamaño de la muestra que se tomo fue de $3 \mathrm{~kg}$; la recolecta se realizó tres tomas de muestras en diferentes puntos del lugar en estudio.

\section{e) Variables medidas}

Los diferentes componentes nutricionales evaluados, se ha trabajado en base a metodologías según Association of Official Analytial Chemists. AOAC (2005).

\section{Humedad}

Se determinó por el método de secado en una estufa al vacío a $105^{\circ} \mathrm{C}$, por un periodo de $12-24$ horas (hasta un peso constante) (método 701.02023) según la AOAC (2005).

\section{Proteína Bruta (PB)}

Se determinó mediante el método de Kjedlhal automático, el cual comprende tres fases: digestión, destilación y titulación, obteniendo como resultado final la cantidad de nitrógeno total (método 711.02-173) según la AOAC (2005).

\section{Extracto etéreo (EE)}

Se determinó por el método de extracción con solvente orgánico mediante el método Soxhlet (método 701.02-031) según la AOAC (2005).

\section{Fibra cruda (FC)}

Se obtuvo mediante la eliminación de los carbohidratos solubles por hidrolisis a compuestos más simples (Azucares), mediante la acción de los ácidos y álcalis en caliente (método 701.03-018) según la AOAC (2005).

\section{Ceniza (CZA)}

Se determinó, mediante la eliminación de materia orgánica por calcinación a $550^{\circ} \mathrm{C}$ por 7 horas (método 702.02-2011) según la AOAC (2005).

\section{Extracto libre de nitrógeno (ELN)}

Obtenida por diferencia, alrededor de $100 \mathrm{del}$ resultado de: humedad, ceniza, extracto etéreo, fibra cruda y proteína cruda (método 923.03) según AOAC (2005).

\section{Fibra detergente Neutra (FDN)}

Se obtuvo mediante la separación de componentes nutricionales solubles de los que no son aprovechables.

Se determinó el grado de digestibilidad de las fibras, en el alimento la muestra fue digerida en una solución de cetil-trimetil-amonio y ácido sulfúrico, el residuo se consideró como la fibra no digerible (método 2002.04), AOAC (2005).

\section{Fibra detergente Acida (FDA)}

Se obtuvo mediante la separación de componentes nutricionales solubles de los que no son aprovechables. 
Se determinó el grado de digestibilidad de las fibras, en el alimento la muestra fue digerida en una solución de acetil-trimetil-amonio y ácido sulfúrico y el residuo se consideró como la fibra no digerible (método 973.18-17) según la AOAC (2005).

Energía calorífica: Se obtuvo mediante la bomba calorimétrica: Para determinar el poder calórico, se utilizó el calorímetro Isoperibólico 6200, modelo 6200 estilo 1108 PARR

Calorimeter. País de fabricación USA.

\section{Digestibilidad in-vitro}

Se obtuvo mediante la medición del grado de aprovechamiento de un alimento y la facilidad con que es convertido en el aparato digestivo en sustancias adecuadas para la absorción, que comprenden dos procesos, digestión y digestibilidad, en soluciones de fosfato de potasio monobásico, sulfato de magnesio heptahidratado, cloruro de sodio, cloruro de calcio hidratado, urea, carbonato de sodio y sulfato de sodio en liquido ruminal. (Protocolo de Ankontechnology incubadora Daisy II D20.

\section{Análisis estadísticos}

Se evaluaron las características nutricionales y digestibilidad de pastos naturalizados de la región Amazonas. En esta investigación se trabajó con un análisis estadístico descriptivo, con tres repeticiones $(n=3)$ de cada pasto. Se empleó el Diseño Completamente al Azar DCA, y la prueba de promedios de Tukey ( $\alpha=0.05$ ). Los datos fueron procesados $\mathrm{y}$ analizados mediante el programa estadístico Statistix V.8.

\section{RESULTADOS}

Las recolecciones de las muestras se realizaron en diferentes lugares de la provincia, teniendo en cuenta un estudio de la línea de base se recolectaron de los lugares más representativos; como se muestran en la tabla 01,02.

.


Tabla 01. Composición nutricional de las gramíneas

\begin{tabular}{|c|c|c|c|c|c|c|c|c|c|c|c|c|c|}
\hline N. común & $\mathrm{MS} \%$ & $\mathrm{HD} \%$ & $\mathrm{~PB} \%$ & $\mathrm{EE} \%$ & FC $\%$ & $\mathrm{CZA} \%$ & ELN\% $\%$ & FDN\% & FDA $\%$ & $\mathrm{P} \%$ & $\mathrm{Ca} \%$ & Kcal EB\% & DIG\% \\
\hline Zacate & 33,79 & $66,21 \pm 0.66^{\circ}$ & $3,71 \pm 0,56^{\mathrm{b}}$ & $2,43 \pm 0,16^{\mathrm{a}}$ & $24,73 \pm 9,87^{\mathrm{a}}$ & $7,49 \pm 1.17^{\mathrm{a}}$ & $55,43 \pm 8,72^{\mathrm{a}}$ & $67,78 \pm 1,57^{\mathrm{a}}$ & $39,72 \pm 3,20^{\mathrm{a}}$ & $0,25 \pm 0,05^{\mathrm{a}}$ & $0,45 \pm 0,03^{\mathrm{a}}$ & $\begin{array}{c}4225,63 \pm 21 \\
0,02^{\mathrm{a}}\end{array}$ & $\underset{\mathrm{b}}{66,35 \pm 11,07}$ \\
\hline Grama azul & 19,9 & $80,1 \pm 0.75^{\mathrm{bc}}$ & $8,30 \pm 2,54^{\text {ab }}$ & $2,05 \pm 1,12^{\mathrm{a}}$ & $27,11 \pm 1,82^{\mathrm{a}}$ & $7,70 \pm 0.57^{\mathrm{a}}$ & $47,71 \pm 3,87^{a}$ & $\underset{\mathrm{b}}{62,75 \pm 0,96^{\mathrm{a}}}$ & $35,51 \pm 3,68^{\mathrm{a}}$ & $0,28 \pm 0,04^{\mathrm{a}}$ & $0,46 \pm 0,04^{\mathrm{a}}$ & $\begin{array}{c}4174,73 \pm 59 \\
63^{\mathrm{a}}\end{array}$ & $66,88 \pm 3,55^{\mathrm{a}}$ \\
\hline Maicillo & 59,2 & $40,8 \pm 0.18^{b}$ & $7,72 \pm 2,19^{\text {ab }}$ & $1,59 \pm 0,41^{a}$ & $26,62 \pm 0,91^{\mathrm{a}}$ & $6,56 \pm 0.82^{a}$ & $49,14 \pm 3,28^{a}$ & $\underset{\mathrm{b}}{59,21+4,49^{\mathrm{a}}}$ & $35,64 \pm 2,03^{\mathrm{a}}$ & $0,14 \pm 0,04^{a}$ & $0,49 \pm 0,30^{\mathrm{a}}$ & $\begin{array}{c}4082,33 \pm 12 \\
2,80^{\mathrm{a}}\end{array}$ & $69,88 \pm 4,71^{a}$ \\
\hline Yuyo & 44,2 & $55.8 \pm 0.65^{\text {be }}$ & $10,91 \pm 0,52^{\mathrm{a}}$ & $3,27 \pm 1,07^{\mathrm{a}}$ & $26,91 \pm 0,70^{\mathrm{a}}$ & $6,81 \pm 2.01^{\mathrm{a}}$ & $45,50 \pm 2,88^{\mathrm{a}}$ & $\underset{\mathrm{b}}{63,95 \pm 5,03^{\mathrm{a}}}$ & $38,36 \pm 1,99^{\mathrm{a}}$ & $0,13 \pm 0,03^{a}$ & $0,28 \pm 0,13^{\mathrm{a}}$ & $\begin{array}{c}4469,18 \pm 17 \\
1,80^{\mathrm{a}}\end{array}$ & $42,36 \pm 4,29^{a}$ \\
\hline Ovillo & 17,1 & $82.9 \pm 1,01^{a}$ & $9,58 \pm 1,53^{\text {bb }}$ & $3,13 \pm 0,68^{a}$ & $22,96 \pm 3,92^{\mathrm{a}}$ & $7,88 \pm 1.40^{\mathrm{a}}$ & $45,66 \pm 2,88^{\mathrm{a}}$ & $55,93 \pm 3,46^{b}$ & $33,93 \pm 1,72^{\mathrm{a}}$ & $0,20 \pm 0,06^{\mathrm{a}}$ & $0,51 \pm 0,09^{\mathrm{a}}$ & $\begin{array}{c}4052,93 \pm 11 \\
2,76^{\mathrm{a}}\end{array}$ & $79,74 \pm 5,10^{a}$ \\
\hline Nudillo & 18,4 & $81,6 \pm 0,66^{b}$ & $10,52 \pm 3,92^{\mathrm{a}}$ & $2,58 \pm 1,28^{\mathrm{a}}$ & $26,86 \pm 1,27^{\mathrm{a}}$ & $7,64 \pm 1,49^{\mathrm{a}}$ & $44,05 \pm 6,01^{\mathrm{a}}$ & $55,69 \pm 2,91^{b}$ & $37,21 \pm 4,73^{\mathrm{a}}$ & $0,33 \pm 0,23^{\mathrm{a}}$ & $0,43 \pm 0,11^{\mathrm{a}}$ & $\begin{array}{c}4109,50 \pm 19 \\
9,71^{2}\end{array}$ & $\underset{\mathrm{a}}{68,07 \pm 14,68}$ \\
\hline Nivel sig. & & 0 & 0,02 & 0,24 & 0,82 & 0,78 & 0,12 & 0,001 & 0,25 & 0,18 & 0,42 & 0,06 & 0,001 \\
\hline Tukey & & $* *$ & * & NS & NS & NS & NS & $* *$ & NS & NS & NS & NS & $* *$ \\
\hline
\end{tabular}

a, b, c: Columnas con letras diferentes, muestran diferencia significativa (Tukey $\alpha=0.05) ;(*)$ diferencia estadísticamente significativa $(\mathrm{p}<0.05) ;(* *)$ diferencia altamente significativa $(\mathrm{p}<0.01)$.

Tabla 02. Composición nutricional de leguminosas y hojas de sub productos agrícolas

\begin{tabular}{|c|c|c|c|c|c|c|c|c|c|c|c|c|c|}
\hline N. común & MS & $\mathrm{Hd} \%$ & $\mathrm{~PB} \%(* *)$ & $\mathrm{EE} \%$ & $\mathrm{FC} \%(* *)$ & $\mathrm{Cza}^{2} \%$ & ELN\% & FDN\% $(* *)$ & FDA\% $\%$ & $\mathrm{P} \%$ & $\mathrm{Ca} \%$ & Kcal EB\% & DIG\% \\
\hline Hoja de yuca & 25 & $75^{ \pm} 0,94^{\mathrm{a}}$ & $23,03 \pm 2,35^{\mathrm{ab}}$ & $7,24 \pm_{1,39^{a}}$ & $15,09 \pm_{2,89} \mathrm{bc}$ & $6,80 \pm_{1,86}{ }^{\mathrm{cd}}$ & $41,08 \pm_{2,72^{\mathrm{a}}}$ & $26,58 \pm 2,73^{\mathrm{a}}$ & $24,62 \pm 3,71^{\mathrm{a}}$ & $0,32 \pm_{0,03}{ }^{\mathrm{b}}$ & $1,47 \pm 331,98^{\mathrm{bcd}}$ & $4780,77 \pm 5,87^{\mathrm{a}}$ & $76,76 \pm 1,01{ }^{b c}$ \\
\hline Hoja de Mostaza & 17,7 & $82,3 \pm_{2,01} \mathrm{a}^{\mathrm{a}}$ & $25,10^{ \pm, 07^{\mathrm{a}}}$ & $3,19 \pm_{0,38} \mathrm{~b}$ & $12,43 \pm_{3,36}{ }^{\mathrm{bc}}$ & $13,33 \pm_{1,78}{ }^{\mathrm{ab}}$ & $37,33 \pm_{6,26}{ }^{\mathrm{a}}$ & $22,08 \pm_{1,22}^{\mathrm{b}}$ & $21,74 \pm 7,28^{\mathrm{a}}$ & $0,3 \pm_{0,10^{\mathrm{b}}}$ & $2,68 \pm 253,26^{\mathrm{ab}}$ & $3835,67 \pm_{4,46^{\mathrm{C}}}^{\mathrm{c}}$ & $91,21 \pm_{0,60}{ }^{\mathrm{a}}$ \\
\hline Alfalfilla & 17,6 & $82,4 \pm_{1,00^{a}}$ & $20,42 \pm_{0,59}{ }^{\mathrm{ab}}$ & $2,81 \pm_{0,14} \mathrm{~b}$ & $23,83 \pm_{10,53}{ }^{\mathrm{abc}}$ & $9,57 \pm_{0,97} \mathrm{bc}$ & $32,54 \pm_{11,12^{\mathrm{a}}}$ & $56,72 \pm_{2,69}{ }^{\mathrm{ab}}$ & $32,41 \pm_{0,78}{ }^{\mathrm{a}}$ & $0,17 \pm_{0,01} \mathrm{~b}^{\mathrm{b}}$ & $2,09 \pm_{165,03}{ }^{\mathrm{cd}}$ & $4141,59 \pm_{4,17} \mathrm{bc}$ & $87,19 \pm_{0,05^{\mathrm{ab}}}$ \\
\hline Trebolillo & 20,8 & $79,2 \pm_{0,68}{ }^{\mathrm{a}}$ & $19,52 \pm_{1,66}{ }^{\mathrm{ab}}$ & $2,16 \pm_{0,66}{ }^{\mathrm{b}}$ & $17,76^{ \pm} \pm_{1,60} \mathrm{abc}$ & $10,65 \pm_{0,96}{ }^{b c}$ & $43,13 \pm_{1,41}{ }^{\mathrm{a}}$ & $52,35 \pm_{5,05^{\mathrm{ab}}}$ & $35,83 \pm_{0,94} 4^{\mathrm{a}}$ & $0,20 \pm_{0,03}{ }^{\mathrm{b}}$ & $1,21 \pm_{165,31}{ }^{\mathrm{cd}}$ & $4431,63 \pm_{4,25} \mathrm{bc}$ & $83,28 \pm_{0,02}{ }^{\mathrm{ab}}$ \\
\hline Retama & 16 & ${ }_{84} \pm_{2,64^{\mathrm{a}}}$ & $10,60 \pm_{3,58} \mathrm{~b}$ & $2,69 \pm_{0,52}{ }^{\mathrm{b}}$ & $30,41 \pm_{4,25}{ }^{\mathrm{a}}$ & $5,14 \pm_{1,89}{ }^{\mathrm{d}}$ & $38,29 \pm_{0,75^{\mathrm{a}}}$ & $54,06 \pm_{8,36}{ }^{\mathrm{ab}}$ & $41,64 \pm_{6,78^{a}}$ & $0,21 \pm_{0,09}{ }^{\mathrm{b}}$ & $1,19 \pm_{131,12}{ }^{\mathrm{cd}}$ & $4592,73 \pm_{1,79}{ }^{\mathrm{ab}}$ & $68,46 \pm_{0,04} \mathrm{c}$ \\
\hline Lengua de vaca & 15,4 & $84,6 \pm 3,40^{\mathrm{a}}$ & $26,43 \pm_{1,14^{a}}$ & $2,26 \pm_{0,63}{ }^{b}$ & $10,26 \pm_{1,66}{ }^{\mathrm{c}}$ & $10,07 \pm_{1,50} \mathrm{~cd}$ & $43,05 \pm_{4,76}{ }^{\mathrm{a}}$ & $35,77 \pm_{18,41} \mathrm{ab}$ & $30,11^{ \pm} 13,95^{\mathrm{a}}$ & $0,30_{0,06}^{\mathrm{b}}$ & $0,51_{83,86}{ }^{\mathrm{d}}$ & $4182,23 \pm 7,61$ & $74,86^{ \pm} \pm_{0,16} \mathrm{bc}$ \\
\hline Siso & 8 & ${ }_{92} \pm_{3,02}{ }^{\mathrm{a}}$ & $15,44 \pm_{4,86}{ }^{\mathrm{b}}$ & $2,11^{ \pm_{0,57}}{ }^{\mathrm{b}}$ & $16,23 \pm_{0,47} \mathrm{bc}$ & $15,53 \pm_{1,73^{\mathrm{a}}}$ & $40,80_{7,31}^{\mathrm{a}}$ & $35,44 \pm_{5,50}{ }^{\mathrm{ab}}$ & $29,25 \pm_{6,91^{a}}$ & ${ }_{0,55} \pm_{0,01}{ }^{\mathrm{a}}$ & $3,36_{177,48^{\mathrm{a}}}$ & $3763,98^{ \pm}, 12^{\mathrm{c}}$ & $84,47 \pm_{0,13}{ }^{\mathrm{ab}}$ \\
\hline Nivel de Sig. & & 0,2 & 0,001 & 0 & 0,001 & 0 & 0,2 & 0,001 & 0,06 & 0 & 0 & 0,001 & 0,001 \\
\hline Tukey & & NS & $* *$ & ** & ** & ** & NS & ** & NS & ** & $* *$ & ** & ** \\
\hline
\end{tabular}

a, b, c: Columnas con letras diferentes, muestran diferencia significativa (Tukey $\alpha=0.05) ;(*)$ diferencia estadísticamente significativa $(\mathrm{p}<0.05) ;(* *)$ dife altamente significativa $(\mathrm{p}<0.01)$ 


\section{DISCUSIÓN}

Los resultados de análisis bromatológico de zacate en la presente evaluación fueron de $3,71 \% \mathrm{~PB} ; 24,73 \%$ FC y $7,49 \%$ Cza, mostrando diferencias con Kawuas et al, (2008), quienes reportaron niveles de 12 PB\%; 25,9 FC\%; 15,3 $\mathrm{Cza} \%$, cuyas diferencias se deben posiblemente a edades, metodologías de análisis y condiciones ambientales diferentes.

Los resultados de análisis bromatológico de la grama azul en la presente evaluación fueron de $8.30 \%$ de $\mathrm{PB} ; 2,05 \%$ de EE; $27,11 \%$ de $\mathrm{FC}$; $47,71 \%$ de ELN; $7,70 \%$ de Cza; $66,88 \%$ de DIG. Mostrando diferencias con Chicco, (1962); quien reporta niveles de $11,86 \%$ de PC; $1,09 \%$ de EE; $27,13 \%$ de FC, $45,34 \%$ de ELN, 7,2\% de Cza; $60,48 \%$ de DIG. Cuyas diferencias se deben posiblemente a edades, metodologías de análisis $\mathrm{y}$ condiciones ambientales diferentes.

Los resultados de análisis bromatológico del maicillo en la presente evaluación fueron de $26.62 \% \mathrm{FC} ; 7,72 \% \mathrm{PC}, 1,59 \% \mathrm{EE}, 6,56 \% \mathrm{CZA}$ 49,14\%ELN. Mostrando diferencias con Chávez, (2008); quien reporta niveles de $15,79 \% \mathrm{FC}$; 8,70\%PC; $2,19 \% \mathrm{EE} ; 6,54 \% \mathrm{Cza} ; 49,14 \% \mathrm{ELN}$. Cuyas diferencias se deben posiblemente a edades, metodologías de análisis y condiciones ambientales diferentes.

Los resultados de análisis bromatológico del yuyo en la presente evaluación fueron de $10,91 \% \mathrm{PC} ; \quad 93,41 \% \mathrm{H} ; \quad 63,95 \% \mathrm{FDN}$; 38,36\%FDA; 6,81\%Cza. Mostrando diferencias con Dueñas et al, (2009); quien reporta niveles de $17,2 \% \mathrm{PC} ; 66,5 \%$ de $\mathrm{H}^{*} ; 59,9 \%$ FDN, 49,9\%FDA; 5,5\%Cza. Cuyas diferencias se deben posiblemente a edades, metodologías de análisis y condiciones ambientales diferentes.
Los resultados de análisis bromatológico del ovillo en la presente evaluación fueron de 10,52\%PC; 79,74\%DIG. Mostrando diferencias con Maza, (2015); quien reporta niveles de $14 \%$ PC y $70 \%$ DIG. Cuyas diferencias se deben posiblemente a edades, metodologías de análisis $\mathrm{y}$ condiciones ambientales diferentes.

Los resultados de análisis bromatológico del nudillo en la presente evaluación fueron de $10,52 \% \mathrm{PC} ; 2,58 \% \mathrm{EE} ; 26,86 \% \mathrm{FC} ; 7,64 \% \mathrm{Cza}$; 44,05\%ELN; 55,69\%FDN; 37,21\%FDA, 0,33\% $\mathrm{P}, 0,43 \% \mathrm{Ca}$; con el nudillo se compara con otras gramíneas que crecen a las mismas condiciones climáticas; tales como, Velázquez, (2009), en su investigación de Rye grass bianual encontró $14,99 \% \mathrm{~PB} ; 1,83 \% \mathrm{EE} ; 24,61 \% \mathrm{FC} ; 11,90 \% \mathrm{Cza}$; 46,66\%ELN; 46,98\%FDN; 34,05\%FDA.

Así mismo, Correa, (2011) en su investigación sobre Kikuyo encontró $20,5 \% \mathrm{~PB} ; 3,63 \% \mathrm{EE}$; $10,6 \% \mathrm{Cza}, \quad 58,1 \% \mathrm{FDN} ; \quad 30,3 \% \mathrm{FDA} ; \quad 0,46 \% \mathrm{P}$; $0,32 \% \mathrm{Ca}$. En comparación con ambas gramíneas diferentes el nudillo presenta diferencias estadísticas entre ambos análisis.

Los resultados de análisis bromatológico de la hoja de yuca en la presente evaluación fueron de $3835,67 \% \mathrm{~EB} ; \quad 25,10 \% \mathrm{PC} ; \quad 15,09 \% \quad \mathrm{FC}$; 7,24\%EE; $21,74 \%$ FDA; $1,47 \% \mathrm{Ca}, 0,32 \%$ P. Mostrando diferencias con Pérez y Yépez, (2009); quienes reportan niveles de $3800 \%$ de EB; $22,70 \% \mathrm{PC} ; 11 \% \mathrm{FC} ; 6,30 \% \mathrm{EE} ; 19,2 \% \mathrm{FDA}$; $1,68 \% \mathrm{Ca}, 0,29 \% \mathrm{P}$. Cuyas diferencias se deben posiblemente a edades, metodologías de análisis y condiciones ambientales diferentes.

Los resultados de análisis bromatológico de la hoja de mostaza en a la presente evaluación fueron de $25,10 \% \mathrm{~PB} ; 22,8 \% \mathrm{FDN} ; 21,74 \% \mathrm{FDA}$; 
2,68\% $\mathrm{Ca} ; \quad 0,33 \% \mathrm{P} ; \quad 3835,67 \% \mathrm{~EB}$. Mostrando diferencias con Antillao, (2009); quienes reportan niveles de $19 \% \mathrm{~PB} ; 17,3 \% \mathrm{FDN}$; 19,2\%FDA; 2,4\%Ca; 0,37\%P; 3800\%EB. Cuyas diferencias se deben posiblemente a edades, metodologías de análisis y condiciones ambientales diferentes.

Los resultados de análisis bromatológico de la alfalfilla en la presente evaluación fueron de 20,42\%PB; $\quad 56,72 \% \mathrm{FDN} ; \quad 32,41 \% \mathrm{FDA}$; 87,19\%DIG. Mostrando diferencias López et al., 2016; quien reporta niveles de $31,5 \% \mathrm{~PB}$; 23,4\%FDN; 14,1\%FDA; 78\%DIG. Cuyas diferencias se deben posiblemente a edades, metodologías de análisis y condiciones ambientales diferentes.

Los resultados de análisis bromatológico del trebolillo en la presente evaluación fueron de $19,52 \% \mathrm{~PB} ; \quad 17,76 \% \mathrm{FC} ; \quad 0,20 \% \mathrm{P} ; \quad 1,21 \% \mathrm{Ca}$. Mostrando diferencias con Tekeli, (2006); reporta niveles de $21,95 \% \mathrm{~PB} ; 15,83 \% \mathrm{FC}$; 0,49\% P; 1,23\%Ca. Así mismo Oliva et al., 2015; reporta 23,29\%PB; 13,34\%FC. Cuyas diferencias se deben posiblemente a edades, metodologías de análisis y condiciones ambientales diferentes.

Los resultados de análisis bromatológico de la retama en la presente evaluación fueron de 10,60\%PC; 2,69\%EE; 68,46\%DIG. Mostrando diferencias con Aguirre, (2008); reporta niveles de $16 ; 9 \% \mathrm{PC} ; \quad 4,9 \% \mathrm{EE}, \quad 11,8 \%$ DIG. Cuyas diferencias se deben posiblemente a edades, metodologías de análisis y condiciones ambientales diferentes.

Los resultados de análisis bromatológico de la lengua de vaca en la presente evaluación fueron
$26,43 \mathrm{~PB} ; \quad 2.26 \% \mathrm{EE} ; \quad 31,5 \% \mathrm{FDN} \quad 0.30 \% \mathrm{P}$, 0.51\%Ca. Mostrando diferencia con Hernán, (2012) en sus investigaciones realizadas reporta niveles de15,4\%PB; $2,81 \% \mathrm{EE} \quad 31,5 \% \mathrm{FDN}$, $0,35 \%$ de $\mathrm{P}, 0,36 \%$ de $\mathrm{Ca}$. Cuyas diferencias se deben posiblemente a edades, metodologías de análisis y condiciones ambientales diferentes.

Los resultados de análisis bromatológicos del siso en la presente evaluación fueron de $15,44 \%$ de PC, $16,23 \%$ de FC, $84,47 \%$ de DIG. Mostrando diferencias con, Oliva et al, (2015); reporta niveles de $19,9 \%$ de $\mathrm{PC}, 13,43 \%$ de FC, $27,8 \%$ de DIG. Cuyas diferencias se deben posiblemente a edades, metodologías de análisis y condiciones ambientales diferentes.

\section{CONCLUSIONES}

Se ha determinado la composición química de 6 variedades de gramíneas, 5 variedades de leguminosas y 2 variedades de hojas de subproductos agrícolas, encontrándose que el Zacate contiene mayores porcentajes en MS (33,6\%); ELN (55,43\%); FDN (67,78\%); Y FDA $(39,72 \%)$, mientras que el Yuyo resalta por su alto contenido de PB (10,91\%); EE (3,27\%) y EB $(4469,18 \mathrm{Kcal})$; dentro del grupo de las gramíneas.

En el grupo de las leguminosas y hojas de sub productos agrícolas, la Hoja de Yuca es la que contiene mayor porcentaje de $\mathrm{MS}(25 \%)$; $\mathrm{EB}(4780,77 \mathrm{Kcal})$ y $\mathrm{EE}(7,24 \%)$, mientras que la Lengua de Vaca es la que contiene mayor contenido de $\mathrm{PB}(26,43 \%)$, mientras que en FC( $30,41 \%)$ y FDA $(41,64 \%)$ es la Retama la que contiene los mayores porcentajes en su composición nutricional. 


\section{REFERENCIA BIBLIOGRÁFICA}

Brack., A. 2004. Biodiversidad y alimentación en el Perú. Universidad San Martín de Porras. Lima. Perú. 17p

Aguirre, J. 2008. Determinación de la composición química y el valor de energía digestible a partir de las pruebas de digestibilidad en alimentos para cuyes. Tesis de grado para optar el grado de ingeniero zootecnista. Facultad de ciencias pecuarias, escuela de ingeniería zootecnista; Escuela Superior Politécnica de Chimborazo. Riobamba. Ecuador. 86p.

Antillao, I. 2009. Utilización de nabo forrajero (Brassica rapa) como suplemento de otoño para la engorda de corderos, en la zona intermedia de Aysén. Tesis para optar el título de ingeniero agrónomo. Escuela de agronomía. Universidad Austral de Chile, Valdivia, Chile. 54p.

Correa, H., 2011. Efecto del manejo del pastoreo y la suplementación alimenticia en vacas lactantes de sistemas especializadas sobre su metabolismo energético y proteico y el contenido de proteína en la leche. Tesis para optar el grado de Doctor en ciencias de la producción animal. Facultad de medicina veterinaria y zootecnia. Bogotá. Colombia. 234p.

Chicco, C.1962. Estudio de la digestibilidad de los pastos en Venezuela.IV. valor nutritivo del pasto pangola (Digitariadecumbens) en varios estados de crecimiento. Revista científica de agronomía tropical. 12(2): (1-3).
Dueñas, F., Benavides, E., \& Rodríguez, A. 2009. Estudio bromatológico y de digestibilidad in situ de la gramínea Chusque (Chusqueascandens) a diferentes edades de cortes. 7(1): 43-54

Flores, E., Cruz, J.,\&Ñaupari, J. 2008. Utilización de praderas cultivadas en secano y praderas naturales para la producción lechera. Disponible www.incagro.gob.pe.

Genin, D \&Alzérreca, H. 2006. Campos nativos de pastoreo y producción animal en la puna semiárida y árida andina. John LibbeyEurotext. 17(1): 1-3

Kawuas, J; Hernández, C; Garza, F; Ibarra, H \& Moreno, G. 2008. Composicion química de cuatro zacates en dos estados fenológicos. Disponible en jkawas@ccr.dsi.uanl.mx.

Oliva, M; Oliva, C; Rojas, D; Oliva, M \& Morales, A. 2015. Identificación botánica de especies nativas de pastos más importantes de las cuencas lecheras de Molinopampa, Pomacochas, y Leymebamba, Amazonas Perú. Scientia Agropecuaria. 6(2): 1-5

Pérez, C \& Yépez, Á. 2009. Suplementación con yuca y follaje de yuca (manihotesculentacrantz) en ganado doble propósito en época de verano. Titulo para optar el grado de Zootecnista. Facultad de ciencias agropecuarias, programa de Zootecnia. Universidad de Lasalle, Bogotá. Colombia. 66p. 
Rivera, A; Valer, F; Pérez, J \& Canales, L. 2008.

Manejo de pastos naturales altoandinos, publicado por el programa de adaptación al cambio climático-PACCPerú. Manual técnico N² 2. Lima. Perú. P24.

Rodríguez, N; Flores, A \&Malpartida, E. 1986. Determinación del valor nutritivo de las principales especies de los pastizales naturales de la sierra central. 3(1): 14-15

Tekeli, A \& Ates, E. 2006. Valores nutritivos de diferentes tréboles anuales (Trifolium $S p$ ) en diferentes etapas de crecimiento. Revistacubana de cienciaagrícola 40(1): (97-103)

Vera, J; Edmundo, L; Guillermo, M; Marta, N. (2009). Evaluación de trébol de olor blanco (Melilotusalbusmedick) en suelos salinos con presencia de $\mathrm{Na}$ freática en el oeste de Santiago del estero, Argentina.

Disponible en rdtoII@gmail.com 